\title{
Become Popular in SNS: Tag Recommendation using FolkPopularityRank to Enhance Social Popularity (Demonstration)
}

\author{
Toshihiko Yamasaki, Yiwei Zhang, Jiani Hu, Shumpei Sano, and Kiyoharu Aizawa \\ Department of Information and Communication Engineering, The University of Tokyo \\ yamasaki@ay-lab.org
}

\begin{abstract}
In this demo, we address two emerging yet challenging problems in social media: (1) scoring the text tags in terms of the influence to the numbers of views, comments, and favorite ratings of images and videos on content sharing services, and (2) recommending additional tags to increase such popularity-related numbers. For these purposes, we present a demo using our FolkPopularityRank (FP-Rank) algorithm, which can score and recommend text tags based on their ability to influence the popularity-related numbers. Our experiments using 1,000 photos showed that we can achieve 1.6 times more views than the original tag sets in Flickr just by adding tags recommended by FP-Rank.
\end{abstract}

\section{Introduction}

In image and video sharing services such as Flickr ${ }^{1}$, the numbers of views, comments, and favorites (likes) are shown along with the content to represent its popularity. Hereafter, we refer to such numbers as "social popularity scores" or "social popularity," aligned with the definition in [Yamasaki et $a l ., 2014]$. In social media networks, it has been a critical issue for both individuals and enterprises to obtain high socialpopularity scores since such scores directly reflect how much attention has been paid to certain contents.

Though one might think that quality of the image and video content is one of the most important factors in receiving higher social popularity scores in social networks, it has been shown that the image and video feature does not contribute much in getting higher social popularity scores. Text tags, on the other hand, play a very important role, because users typically locate content via text searches.

In [Yamasaki et al., 2017], we presented a new algorithm called FolkPopularityRank (or FP-Rank). FP-Rank is inspired by PageRank [Brin and Page, 1998], the well-known web page ranking algorithm, and FolkRank [Hotho et al., 2006], PageRank's folksonomy-based descendant that also considers users, resources, and tags. The contribution of this paper is that FP-Rank can recommend tags that enhance social popularity scores. The recommendation is done based on

\footnotetext{
${ }^{1}$ https://www.flickr.com/
}

existing tags, and therefore the recommended tags are related to the content of the image/video. Although numerous tag recommendation systems have already been proposed, they are focused only on tags that are semantically correct for describing the content. To the best of our knowledge, tag recommendations designed to increase social popularity scores is firstly presented.

In our demo, we present a WEB browser-based tag recommendation system for Flickr. Our system recommends tags that can potentially increase the number of views for any image/video in Flickr as long as it is annotated with some tags.

\section{Folk Popularity Rank}

\subsection{Tag Ranking Algorithm}

We would like to emphasize that conventional tag ranking and recommendation systems in social media are designed to add semantically proper tags. On the other hand, we have been proposing an algorithm that can extract tags with a higher level of influence on social popularity scores. As a result, we can recommend tags that enhance social popularity.

The equation for our FP-Rank scores, $\mathbf{s}$, is defined as follows.

$$
\begin{aligned}
\mathbf{r}_{F P} & =\alpha A_{F P} \mathbf{r}_{F P}+(1-\alpha) \mathbf{p}, \\
\mathbf{s} & =\mathbf{r}_{F P}-\mathbf{r}_{F}^{t a g},
\end{aligned}
$$

where $\mathbf{r}_{F}^{t a g}$ is the tag-only FolkRank score, $\alpha$ is a damping factor, and $\mathbf{p}$ is a random surfer component.

There are two fundamental differences from PageRank and FolkRank. The first involves the design of the matrix $A_{F P}$. $A_{F P}$ is a square matrix whose size is $T \times T$, where $T$ is the number of unique tags in the service. The matrix $A_{F P}$ can be interpreted as a combination of two matrices by introducing the tag-content matrices:

$$
A_{F P}=B_{w} \times B_{t}^{T} .
$$

Here, $B_{w}$ and $B_{t}$ are $T \times C$ row-stochastic and columnstochastic matrices, respectively, where $C$ is the number of the images and videos in the service. The $i$ th row vector in $B_{w}$ is a set of social popularity scores for the content tagged with the $\operatorname{tag} i$, normalized by the sum of the scores in the row. The $j$ th column vector in $B_{t}$ is a set of tag usage flags normalized by the total number of tags attached to the $j$ th 


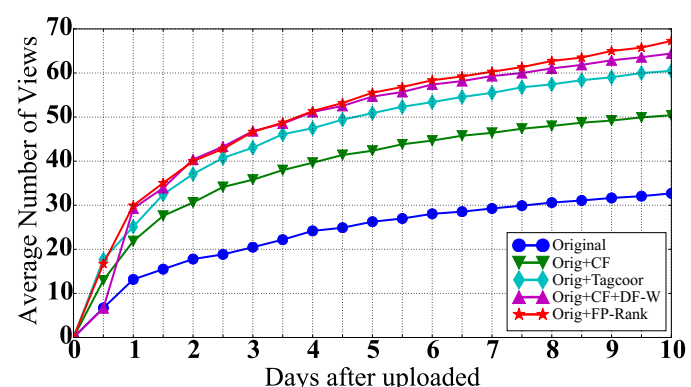

Figure 1: Average number of views per image.

content. As a result, the component of the matrix $A_{F P}, a_{i j}$ is calculated as follows.

$$
a_{i j}=\sum_{d \in \mathbf{D}} \frac{u(d)_{i}}{(\# \text { of tags attached to content } d)},
$$

where $d$ is the index of the image or video content that contains both tags $i$ and $j, \mathbf{D}$ is the training dataset, and $u(d)_{i}$ is the weight for the tag $i$ annotated to the content $d$. FP-Rank calculates $u(d)_{i}$ by considering the content's social popularity scores:

$$
\begin{aligned}
u(d)_{i}= & (\text { current score for tag } i) \times \\
& \frac{(\text { social pop. score of content } d)+1}{\sum(\text { social pop. score of content with tag } i)}(5)
\end{aligned}
$$

where $k$ is a parameter to prevent $u(d)_{i}$ from being 0 .

\subsection{Tag Recommendation}

Similar to FolkRank, tags can be recommended by the following equation.

$$
\mathbf{w}_{F P}=\mathbf{s}^{(d=1)}-\mathbf{s}^{(d=0)},
$$

where $\mathbf{w}_{F P}$ is a weight vector with one entry for each tag. The random surfer component for the already existing tags is set to 1 , and the others are set to $0 \sim 1$; the eq. (1) is iterated until convergence.

\subsection{Experimental results}

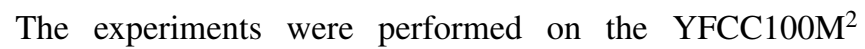
dataset. We randomly selected 60,000 images that have more than 20 tags and over 5000 views of the whole YFCC100m dataset for training and randomly selected 947 images annotated with over 20 tags but different number of views for testing. We compared our FP-Rank with three baselines (please refer to [Yamasaki et al., 2017] for details). We added 10 tags to the original tag sets and uploaded back to Flickr.

The results are shown in Figure 1. FP-Rank performed the best. The average number of views is 2 times higher than that with the original tags. This proves that FP-Rank makes better recommendations with a higher level of influence on popularity boosting over the other three methods.

\footnotetext{
${ }^{2}$ http://webscope.sandbox.yahoo.com/catalog. php? datatype=i\&did=67
}

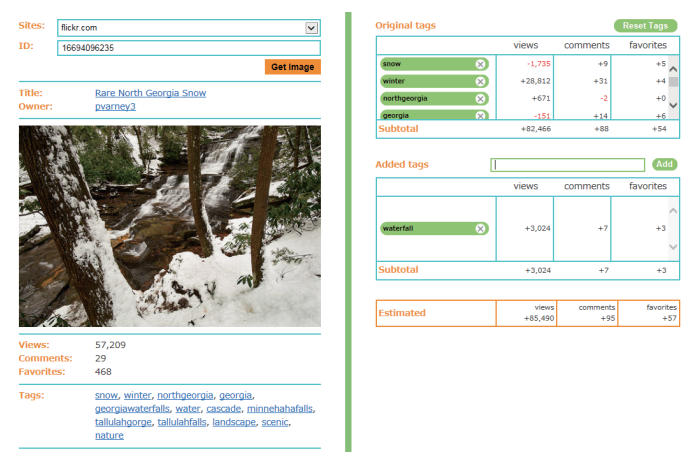

Figure 2: Our web-based demonstration system.

\section{Demo}

Our demo system is demonstrated in Fig. 2. If the content ID is given to the system, the photo/video and its metadata such as owner, title, the number of views, comments, favorites, and original tags are all retrieved on the left.

The first window on the right shows how much each tag contributes to the number of views, comments, and favorites by using our DF-W based regression [Yamasaki et al., 2014]. The second window shows the recommended tags by FPRank [Yamasaki et al., 2017] to enhance the social popularity scores. It is also possible to manually add tags. In this case, keywords are retrieved by forward match, but suggested by the importance order of FP-Rank, not by the alphabetical order. In this manner, users can select proper tags that are influential to social popularity scores. The predicted number of views, comments, and favorites are calculated on the bottom.

\section{Acknowledgments}

This work was partially supported by the Grants-in-Aid for Scientific Research (no. 26700008) from JSPS, JST-CREST (JPMJCR1686), and Microsoft IJARC core13. J. Hu is now with Microsoft Development, Co., Ltd. and S. Sano is now with Yahoo Japan Corporation.

\section{References}

[Brin and Page, 1998] Sergey Brin and Lawrence Page. The anatomy of a large-scale hypertextual web search engine. Computer Networks and ISDN Systems, 30:107-117, 1998.

[Hotho et al., 2006] Andreas Hotho, Robert Jaschke, Christoph Schmitz, and Gerd Stumme. Folkrank: A ranking algorithm for folksonomies. In $L W A$, volume 1 , pages 111-114, 2006.

[Yamasaki et al., 2014] Toshihiko Yamasaki, Shumpei Sano, and Kiyoharu Aizawa. Social popularity score: Predicting numbers of views, comments, and favorites of social photos using only annotations. In WISMM, pages 3-8, 2014.

[Yamasaki et al., 2017] Toshihiko Yamasaki, Jiani Hu, Shumpei Sano, and Kiyoharu Aizawa. Folkpopularityrank: Tag recommendation for enhancing social popularity using text tags in content sharing services. In IJCAI, 2017. 